\section{pIRES-CD4t, a Dicistronic Expression Vector for MACS- or FACS-Based Selection of Transfected Cells}

\author{
BioTechniques 26:683-688 (April 1999)
}

\begin{abstract}
To facilitate the selection of cell lines expressing transfected genes of interest, a plasmid vector has been constructed that directs the co-expression of heterologous cDNAs and a 3'-positioned cassette encoding a truncated CD4 marker. An encephalomyocarditis virus internal ribosomal entry site (IRES) mediates translational initiation from this 3' cassette, and a cytomegalovirus promoter drives dicistronic transcript expression. To test the utility of this vector, a luciferase reporter gene was inserted, and this construct (pIRES-CD4tluc) was electrotransfected into myeloid FDCW2 cells. As monitored by flow cytometry and luciferase assays, three rounds of magnetic cells sorting (MACS) yielded $\geq 90 \%$ CD4t-positive cells with an average density of $17000 \mathrm{CD} 4 \mathrm{t}$ molecules per cell. In ten clonal sublines analyzed, luciferase expression was uniformly high and stable over a test period of three months. Finally, a comparison of MACS-vs. FACS-based isolation of transfected cells showed two to three rapid rounds of MACS to be somewhat more effective. Thus, pIRES-CD4t should prove useful in the direct and rapid selection of relevant stably or transiently transfected cells.
\end{abstract}

\section{INTRODUCTION}

Dicistronic vectors recently have been developed that increase the frequency of heterologous gene expression in transfected cells $(7,10,11,13$, 14). In these vectors, a single promoter drives the transcription of tandem cDNAs, and translation of the 3'-positioned cDNA (typically a selectable marker) is directed by an internal ribosomal entry site (IRES) from encephalomyocarditis virus (EMCV) (5). EMCV possesses an uncapped RNA strand including a 600-nucleotide (nt) IRES with a $5^{\prime}$ untranslated region (UTR) that initiates translation by means of a cap-independent mechanism (5). When positioned between heterologous cDNAs, this element can mediate the translation of $3^{\prime}$-positioned constructs $(7,9)$, thereby coupling the expression to 5 '-inserted cDNAs $(7,11,14)$. This provides an advantage over co-transfected or dual-promoter vectors in which expression is often uncoupled $(7,8)$. Among IRES-containing dicistronic vectors, several distinct $3^{\prime}$ marker cassettes exist, including: pCIN (14) and pMK (11) vectors include neomycin phosphotransferase, the tetracycline inducible vector pTETißgeo (10) encodes a lacZ-neomycin phosphotransferase fusion gene and the retroviral vector pED4 (7) contains a 3 '-positioned dihydrofolate reductase (DHFR) cassette. Tet-inducible vectors such as pTETißgeo, however, require extended induction and incubations with fluorescein di- $\beta$-D-galactopyranoside, while pED4 requires the use of DHFR $^{-/-}$target cells. Dicistronic vectors encoding green fluorescent protein (GFP) (e.g., pTR-DC/GFP; Reference 13) allow selection by fluorescence-activated cell sorting (FACS), but in at least certain systems, clones stably expressing S65T GFP can be inactivated by rearrangement or mutation (3).

For the selection of tranfected cells, immunomagnetic methods (i.e., magnetic cell sorting [MACS]) using ferrit particle-coupled antibodies against cell-surface markers provide a direct approach (12,17). For MACS, pSR $\alpha M S V-T K C D 8$ and pMT-IRESCD4 vectors have been previously developed. However, the pSR $\alpha \mathrm{MSV}$ TKCD8 vector is not dicistronic (6), and in studies of stably transfected cells, pMT-IRES-CD4 was co-transfected with a neomycin-encoding plasmid; it is unclear whether this vector alone can be used to efficiently select stably transfected lines (18). In addition, the use of a full-length CD4 cDNA in this vector is potentially complicating. To provide for the selection of stably transfected cells co-expressing marker and heterologous cDNAs at high frequencies, a vector presently has been developed in which a cytomegalovirus (CMV) promoter drives ex- 
pression of dicistronic transcripts that contain a centrally positioned EMCV IRES and a $3^{\prime}$ cassette encoding a truncated form of human CD4 gene (1). To test the utility of this vector, a luciferase reporter gene was inserted, and this construct was electrotransfected into myeloid FDCW2 cells (16). Cells stably expressing $\mathrm{CD} 4 \mathrm{t}$ were isolated by either MACS or FACS. Analyses of marker and reporter expression show that this pIRES-CD4t vector provides for the direct and efficient isolation of transfected cells and stably drives the coupled expression of inserted heterologous cDNAs.

\section{MATERIALS AND METHODS}

\section{Dicistronic Expression Vectors}

pIRES-CD4t was constructed by replacing the neomycin phosphotransferase gene in pIRES1neo (CLONTECH Laboratories, Palo Alto, CA, USA) with a cDNA encoding the extracellular and transmembrane domains of human CD4 (1). This cDNA was excised from pMACS 4 (Miltenyi Biotec, Bergisch Gladbach, Germany) using EcoRI (site located 81 nt upstream from the AUG start codon) and PstI (site located $12 \mathrm{nt} 3^{\prime}$ to a stop codon positioned five amino acids after the transmembrane domain) and was cloned (1363-bp fragment) into pBluescript ${ }^{\circledR} \mathrm{KS}(+)$ (Stratagene, Palo Alto, CA, USA). pBS-CD4t then was digested with EcoRI, blunt-ended using the Klenow fragment of DNA polymerase I (New England Biolabs, Beverly, MA, USA) and digested with $\mathrm{XbaI}$ to yield a 5'-blunted, 3' XbaI fragment. pIRES1neo was digested with SmaI and $\mathrm{XbaI}$ to excise the neomycin phosphotransferase gene, and the above CD4t cDNA fragment was ligated into these sites to yield pIRES-CD4t. pIRES-CD4t-luc was constructed as follows. A luciferase cDNA was isolated from pSP-luc+ (Promega, Madison, WI, USA) by digestion with HindIII, blunt-ending with the Klenow fragment of DNA polymerase I and digestion with EcoRI. This 1669-bp fragment then was cloned into pIRES-CD4t at EcoRV and EcoRI sites to yield pIRESCD4t-luc.
Table 1. Sustained Expression of Luciferase Activity in Polyclonal MACS 4 FDCW2-pIRES-CD4tluc Cells and Ten Clonal Sublines

\begin{tabular}{|lccc|}
\hline & $\mathbf{6}$ weeks & $\mathbf{8}$ weeks & 12 weeks \\
\hline MACS 4 & $11.48 \pm 5.82$ & $16.69 \pm 2.55$ & $10.95 \pm 1.92$ \\
NonSelected & $0.25 \pm 0.04$ & $0.15 \pm 0.02$ & $0.02 \pm 0.01$ \\
Clone A12 & 19.5 & 13.4 & 17.0 \\
Clone B5 & 19.6 & 8.9 & 7.7 \\
Clone D8 & 24.7 & 32.4 & 21.9 \\
Clone D12 & 26.9 & 15.1 & 22.9 \\
Clone E11 & 28.5 & 24.9 & 21.5 \\
Clone E12 & 14.0 & 29.1 & 10.5 \\
Clone G7 & 8.6 & 10.5 & 9.4 \\
Clone H1 & 26.5 & 14.8 & 24.2 \\
Clone H3 & 17.6 & 17.8 & 15.9 \\
Clone H9 & 15.0 & 8.7 & 18.8 \\
\hline
\end{tabular}

\section{Cell Culture, Electrotransfections and MACS Selection}

FDCW2 cells were maintained at $37^{\circ} \mathrm{C}, 5 \% \mathrm{CO}_{2}$ in OPTI-MEM ${ }^{\mathrm{TM}}$ I medium (Life Technologies, Gaithersburg, MD, USA) supplemented with $8 \%$ fetal bovine serum (FBS) and $8 \%$ Wehi3Bcell-conditioned medium (W3CM) as a source of Interleukin-3 (IL-3). Electrotransfections were performed as detailed previously (16). Following elec- trotransfections, $1 \times 10^{7}$ expanded cells were subjected to selection using antiCD4 conjugated microbeads (Miltenyi Biotec). Cells were collected by centrifugation $(800 \times g$ for $10 \mathrm{~min})$ and were resuspended in $10 \mathrm{~mL}$ of degassed phosphate-buffered saline (PBS) $(2.7$ $\mathrm{mM} \mathrm{KCl}, 140 \mathrm{mM} \mathrm{NaCl}, 1.5 \mathrm{mM}$ $\mathrm{KH}_{2} \mathrm{PO}_{4}, 8 \mathrm{mM} \mathrm{Na} \mathrm{HPO}_{4} \cdot 7 \mathrm{H}_{2} \mathrm{O}, \mathrm{pH}$ 7.2) containing $5 \mathrm{mM} \mathrm{Na} 2 \mathrm{EDTA}$. Cells then were collected, resuspended in 320 $\mu \mathrm{L}$ of PBS, $5 \mathrm{mM} \mathrm{Na} 2$ EDTA, $0.5 \%$

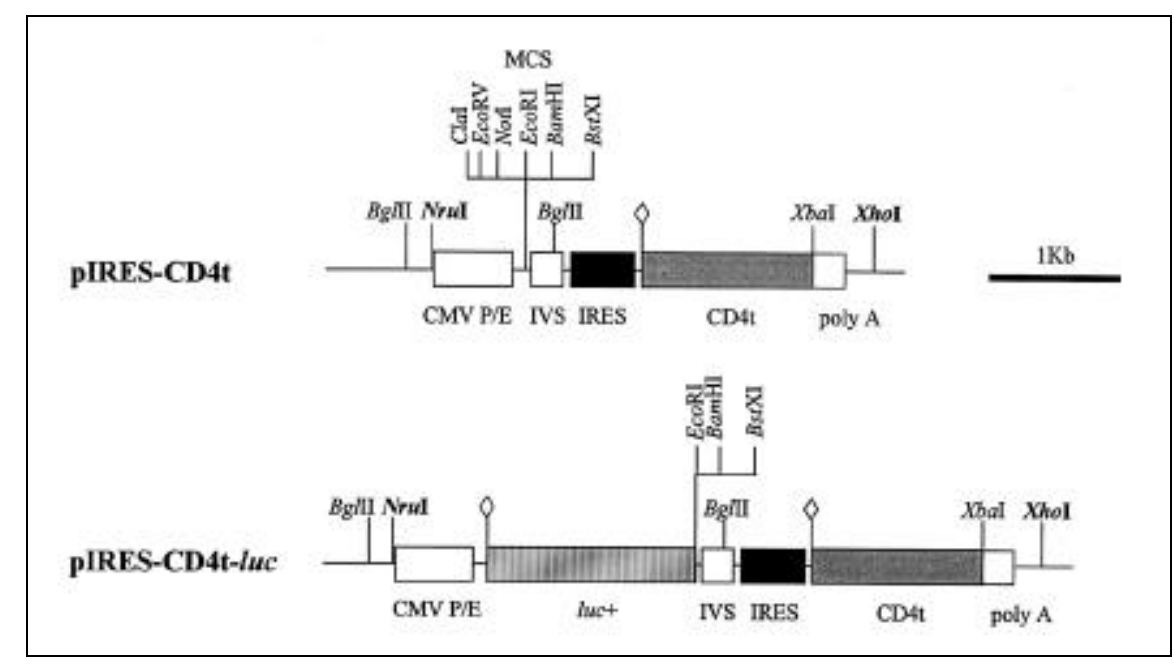

Figure 1. pIRES-CD4t and pIRES-CD4t-luc dicistronic expression vectors. pIRES-CD4t was constructed by cloning a CD4t cDNA from pBS-CD4t (see Materials and Methods) into pIRESneo (originally described as pCIN4; Reference 3). Contained in pIRES-CD4t are a CMV major immediate early enhancer/promoter (CMV P/E), a synthetic intron known to enhance mRNA stability (IVS), an MCS, a 585-bp EMCV IRES, the above 3'-positioned CD4t cDNA and a polyadenylation signal sequence from a bovine growth hormone cDNA (omitted in this schematic are an ampicillin-resistance gene and an E. coli origin of replication). In CD4t, the start codon is located $102 \mathrm{nt}$ downstream of AUG 10 within the EMCV IRES. pIRES-CD4-luc was constructed by cloning a luciferase cDNA from pSP-luc+ into $E c o R V$ and EcoRI sites within the MCS of pIRES-CD4t. Restriction sites that were destroyed during blunt-ended ligation reactions are indexed by open diamonds ( $\diamond)$. Two unique restriction sites, NruI and $X h o I$, available for linearization of the vector are in bold. 


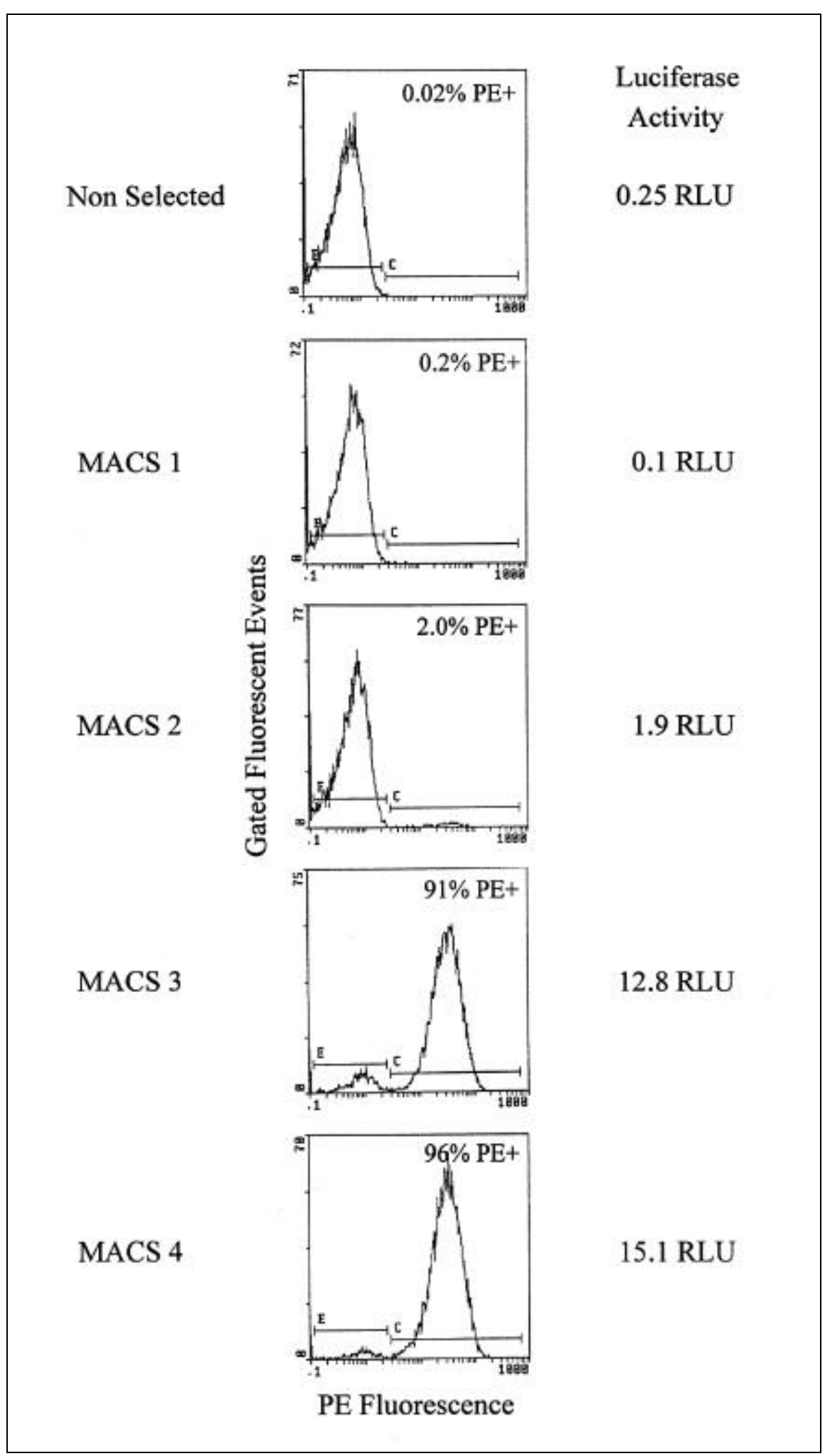

Figure 2. CD4t marker expression and luciferase activity is coupled in MACS-selected FDC2pIRES-CD4t-luc cells. FDCW2 cells electrotransfected with pIRES-CD4t-luc were selected by four rounds of MACS to yield populations MACS 1-4. Each population of cells was analyzed by flow cytometry using a PE-conjugated $\alpha$-CD4 MAb (RPA-T4). Each panel shows histograms of the number of gated fluorescent events (y-axis) vs. PE fluorescence intensity (x-axis). In each histogram, percentages of $\mathrm{PE}-$ positive (PE+) cells (C gate) are indexed in the upper right corner. For each MACS population, luciferase activity is indexed (RLU). Results shown are representative of three independent experiments. 


\section{Short Technical Reports}

BSA and were incubated with $80 \mu \mathrm{L}$ of MACSelect 4 Microbeads (Miltenyi Biotec) for $15 \mathrm{~min}$ at $10^{\circ} \mathrm{C}$. The total sample volume then was adjusted to 2 $\mathrm{mL}$ using PBS, $5 \mathrm{mM} \mathrm{Na}{ }_{2}$ EDTA, 0.5\% BSA and was loaded onto an $\mathrm{MS}^{+} / \mathrm{RS}^{+}$ column within the magnetic field of a miniMACS Separation Unit (Miltenyi Biotec). The flow-through volume was collected and was reapplied to the column. The column then was washed $6 \times$ with $500 \mu \mathrm{L}$ of PBS, $5 \mathrm{mM} \mathrm{Na}{ }_{2}$ EDTA and $0.5 \%$ BSA. Bound cells were eluted in $1 \mathrm{~mL}$ of PBS, $5 \mathrm{mM} \mathrm{Na}{ }_{2}$ EDTA and $0.5 \% \mathrm{BSA}$, collected, resuspended in 3 $\mathrm{mL}$ of OPTI-MEM I medium, $8 \%$ FBS, $8 \% \mathrm{~W} 3 \mathrm{CM}$ and cultured for 2 days in a 6-well Falcon ${ }^{\circledR}$ tissue culture plate (Becton Dickinson Labware, Bedford, MA, USA) in the presence of penicillin $(100 \mathrm{U} / \mathrm{mL})$, streptomycin $(100 \mu \mathrm{g} / \mathrm{mL})$ and amphotericin B $(0.25 \mu \mathrm{g} / \mathrm{mL})$.

\section{Flow Cytometry, FACS and Luciferase Assays}

In assays of CD4t expression, cells were labeled with a phycoerythrin (PE)-conjugated anti-CD4 monoclonal antibody (MAb) (PE-RPA-T4; PharMingen, San Diego, CA, USA) and were analyzed by flow cytometry. Specifically, cells $\left(1 \times 10^{7}\right)$ were incubated at $4^{\circ} \mathrm{C}$ for $10 \mathrm{~min}$, collected by centrifugation at $200 \times g$ for $10 \mathrm{~min}$ and washed at $4^{\circ} \mathrm{C}$ in PBS and $0.05 \%$ BSA. Cells then were collected at $1000 \times g$ for $5 \mathrm{~min}$, resuspended in $1 \mathrm{~mL}$ PBS, $0.05 \%$ BSA containing $200 \mu \mathrm{g}$ of mouse $\gamma$-globulins (Cohn fraction II and III; Sigma, St. Louis, MO, USA) and incubated at $4^{\circ} \mathrm{C}$ for $15 \mathrm{~min}$. PERPA-T4 antibody $(0.5 \mu \mathrm{g})$ was added to $100 \mu \mathrm{L}$ of resuspended cells $\left(1 \times 10^{6}\right.$ cells), and samples were incubated at

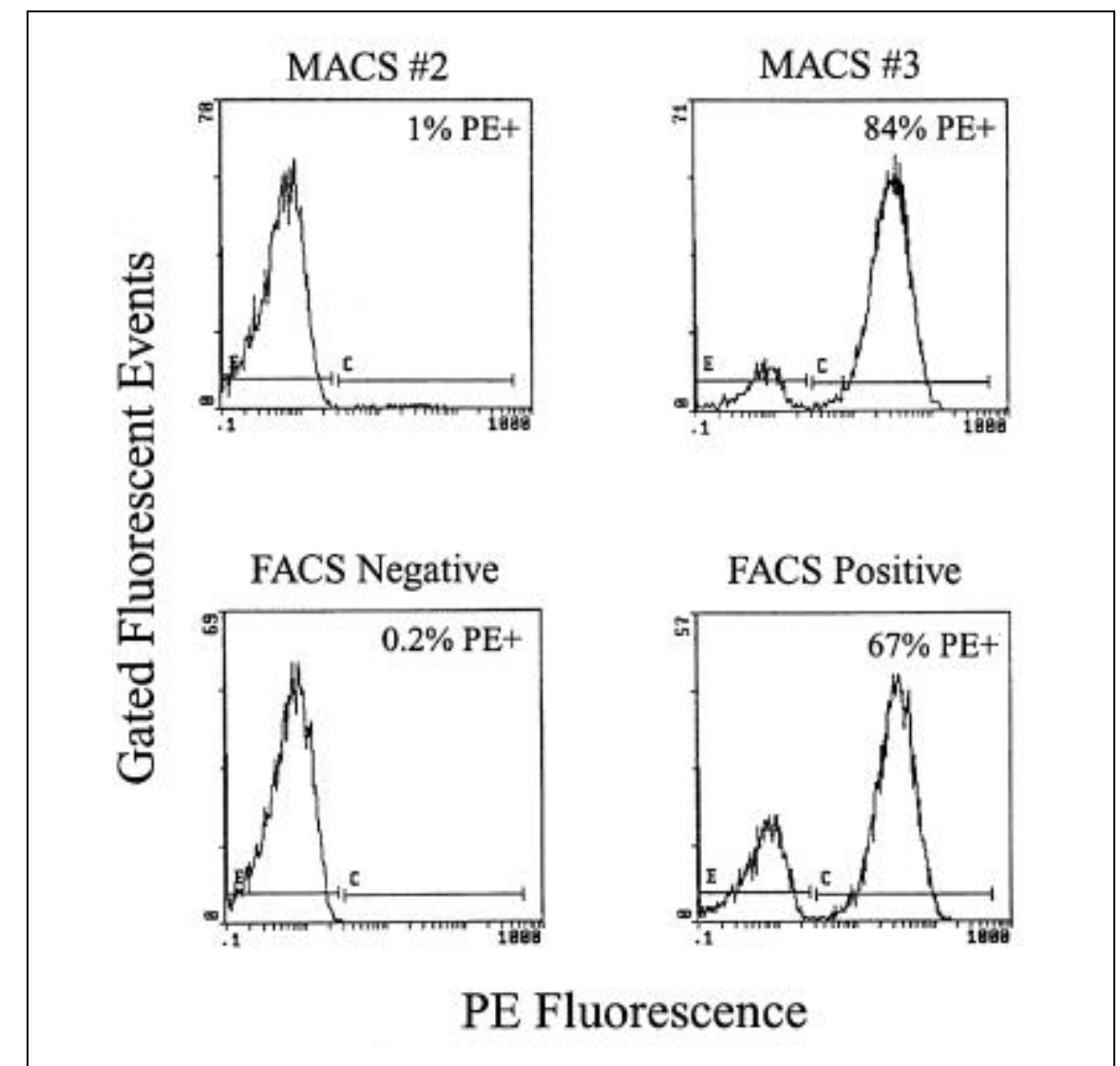

Figure 3. Isolation of FDCW2-pIRES-CD4t-luc cells by FACS. MACS 2 cells (see Figure 2) were stained using optimized conditions with PE-RPA-T4 and positive (vs. negative) populations of cells were isolated by FACS (FACS-negative vs. -positive, lower panels). In parallel, MACS 2 cells were subjected to one round of MACS yielding MACS 3 cells (upper right panel). Shown are histograms of the number of gated fluorescent events (y-axis) vs. the level of PE fluorescence intensity (x-axis) for each FACS- or MACS-selected line. Percentages of CD4t-positive cells (gate C) are indexed in upper right corners of each histogram. 
$4^{\circ} \mathrm{C}$ for $60 \mathrm{~min}$. Cells then were diluted with $1 \mathrm{~mL}$ of PBS, $0.05 \%$ BSA, collected $(1000 \times g$ for $5 \mathrm{~min})$, resuspended in $500 \mu \mathrm{L}$ of PBS, $0.05 \%$ BSA and held at $4^{\circ} \mathrm{C}$. Flow cytometric analyses were performed using an EPICS $^{\circledR}$ XL system (15-mW argon-ion laser, 488 nm) and Coulter ${ }^{\circledR}$ XL-2 software (both from Beckman Coulter, Fullerton, CA, USA). PE-conjugated calibration particles (Catalog No. RCP 30-5; Spherotech, Libertyville, IL, USA) were used to calculate the average number of PERPA-T4 molecules on the surface of transfected cells. FACS was performed using an EPICS Elite system (15-mW argon-ion laser, $488 \mathrm{~nm}$; Beckman Coulter), and PE-positive vs. -negative populations were gated and collected. Each population was washed in OPTIMEM I medium, cultured for $24 \mathrm{~h}$ in OPTI-MEM I medium, 8\% FBS, 8\% W3CM supplemented with penicillin (200 U/mL), streptomycin (200 $\mu \mathrm{g} / \mathrm{mL})$ and amphotericin B (0.5 $\mu \mathrm{g} / \mathrm{mL}$ ) (Sigma) and expanded. In luciferase assays, cell lysates were prepared by collecting cells $\left(3 \times 10^{6}\right)$ at $200 \times g$ for $10 \mathrm{~min}$, washing cells in 10 $\mathrm{mL}$ of PBS and preparing lysates in 60 $\mu \mathrm{L}$ of $1 \%$ Triton ${ }^{\circledR} \mathrm{X}-100,2 \mathrm{mM} 1,2-\mathrm{di}$ aminocyclohexane- $N, N, N^{\prime}, N^{\prime}$-tetraacetic acid, $2 \mathrm{mM}$ dithiothreitol (DTT), 10\% glycerol, $25 \mathrm{mM}$ Tris-phosphate, pH 7.8 (Catalog No. E153A; Promega). To ensure lysis, samples were mixed by vortex mixing for $15 \mathrm{~min}$ at $23^{\circ} \mathrm{C}$, and cleared lysates were prepared by centrifugation at $8000 \times g$ for $10 \mathrm{~min}$. In luciferase assays, $20 \mu \mathrm{L}$ of lysate were added to $100 \mu \mathrm{L}$ of $20 \mathrm{mM}$ tricine, 1.07 $\mathrm{mM}\left(\mathrm{MgCO}_{3}\right)_{4} \mathrm{Mg}(\mathrm{OH})_{2} \cdot 5 \mathrm{H}_{2} 0,2.67$ $\mathrm{mM} \mathrm{MgSO}_{4}, 0.1 \mathrm{mM}$ EDTA, $33.3 \mathrm{mM}$ DTT, $270 \mu \mathrm{M}$ coenzyme A, $470 \mu \mathrm{M}$ luciferin, $530 \mu \mathrm{M}$ ATP (Catalog No. E1483; Promega). To control for possible differences in cell number or lysis, lysates were assayed for protein content (BCA Protein Assay; Pierce Chemical, Rockford, IL, USA), and values were used to normalize luciferase activities.

\section{RESULTS}

To test for coupled expression of CD4t and a cDNA insert, a luciferase gene was cloned into pIRES-CD4t, and this construct (Figure 1) was transfected into FDCW2 cells. By intent, FDCW2 cells were used based on their low transfection efficiency and the associated challenge in retrieving derived cell lines that express transfected cDNAs at useful, uniform levels. Transfected cells were subjected to four rounds of MACS (populations MACS 1-4) and were assayed for CD4t expression (Figure 2). Three rounds of MACS yielded cells expressing CD4t at high frequencies (91\% PE-RPA-T4 antibody positive), while a fourth round of MACS only slightly increased frequencies of CD4t-positive cells (96\%).

Absolute levels of CD4t expression were estimated using PE-conjugated calibration particles. In MACS 4 cells, an average of 34000 PE-RPA-T4 antibodies were bound per cell, and CD4t expression is estimated at 17000 molecules per cell. This indicates efficient translation of CD4t from pIRES-CD4t$l u c$ and predicts that selected cells should uniformly express linked $5^{\prime}$ cDNAs at useful levels. To test this, luciferase expression was assayed in polyclonal FDCW2-pIRES-CD4t-luc cells and in sublines isolated by dilution cloning (16) of MACS 4 cells. Upon MACS retrieval, luciferase activity in polyclonal populations increased markedly (Figure 2). In addition, luciferase expression in each of ten clonal sub-lines was comparable to that in MACS 4 cells (Table 1). This result confirms tightly coupled expression of marker and inserted cDNAs. To test stability of expression, luciferase activity also was monitored over a test period of three months. Activity remained high in all cells tested in the absence of further selection (Table 1).

Finally, MACS and FACS were compared directly as methods for isolating stably transfected cells. Here, a MACS 2 population of FDCW2pIRES-CD4t-luc cells was used based on the presence of low but detectable frequencies of $\mathrm{CD} 4 \mathrm{t}$-expressing cells (approximately 1\%). FACS was applied, and two gated populations of cells (FACS-positive and FACS-negative cells) were recovered. Cells from each population were expanded and analyzed for CD4t and luciferase expression (Figure 3). FACS increased frequencies of CD4t-positive cells to $67 \%$, 
whereas MACS (performed in parallel) increased frequencies to $87 \%$. In addition, FACS-positive and MACS 3 populations each expressed significantly increased levels of luciferase activity compared to the FACS-negative and MACS 2 populations (16.7 and 7.7 vs. 0.5 and 0.4 relative light units [RLU], respectively). Therefore, MACS was at least as efficient as FACS in selecting transfected CD4t-positive cells.

\section{DISCUSSION}

pIRES-CD4t has been developed as an expression vector that combines the advantages of dicistronic expression and MACS. Two features that merit discussion are the choice of CD4t as a marker and the nature of the IRES used to direct translation. Studies of CD4 in human immunodeficiency virus (HIV) infection have led to the development of MAbs with high specificities and affinities as conjugated forms for direct use in MACS and FACS (4). Thus, cells transfected with pIRES-CD4t can be efficiently retrieved and analyzed quantitatively using commercially available reagents. The $\alpha \mathrm{CD} 4$-conjugated, ferritdextran microbeads used in the present study, in fact, have been applied to isolate CD4+ T-cells from peripheral blood lymphocytes in clinical applications (19). Since CD4 expression is restricted largely to developing thymocytes and major histocompatibility complex (MHC) II-restricted mature T-lymphocytes (2), this marker can be used in a wide variety of cells and cell lines.

IRES from EMCV was used since eukaryotic initiation factors eIF-2/2B bind to this IRES with high affinity (15), and because this IRES is perhaps best characterized $(7,9)$. In addition, in pIRES-CD4t, this IRES is engineered to repress the expression of inappropriate fusion proteins, and to attenuate $3^{\prime}$ cassette translation (the predominant initiation site AUG-11 is deleted, and a 94-bp spacer cassette shifts translational initiation to the Met ${ }^{1}$ of CD4t; Reference 14). This modification also attenuates translation of $3^{\prime}$ cassettes within dicistronic transcripts and favors highlevel translation of 5'-positioned cDNAs (14). Thus, pIRES-CD4t is a unique dicistronic vector that provides for the coupled expression of heterologous cDNAs and a $3^{\prime}$-positioned CD4 marker cassette. Transfected cells can be isolated directly by either MACS or FACS, and coordinate expression provides for sustained high frequencies of heterologous cDNA expression in selected cells.

\section{REFERENCES}

1.Bedinger, P., A. Moriarty, R.C. von Borstel II, N.J. Donovan, K.S. Steimer and D.R. Littman. 1988. Internalization of the human immunodeficiency virus does not require the cytoplasmic domain of CD4. Nature 334:162165.

2.Bowers, K., C. Pitcher and M. Marsh. 1997. CD4: a co-receptor in the immune response and HIV infection. Int. J. Biochem. Cell Biol. 29:871-875.

3.Hanazono, Y., J.M. Yu, C.E. Dunbar and R.V.B. Emmons. 1997. Green fluorescent protein retroviral vectors: low titer and high recombination frequency suggest a selective disadvantage. Hum. Gene Ther. 8:1313-1319.

4.Holzer, T.J., C.A. Heynen, R.M. Novak, D.L. Pitrak and G.J. Dawson. 1993. Frequency of cells positive for HIV-1 p24 antigen assessed by flow cytometry. AIDS 7 (Suppl.) 2:S3-S5.

5.Jackson, R.J., M.T. Howell and A. Kaminski. 1990. The novel mechanism of initiation of picarnovirus RNA translation. Trends Biochem. Sci. 15:477-483.

6.Kamijo, T., J.D. Weber, G. Zambetti, F. Zindy, M.F. Roussel and C.J. Sherr. 1998. Functional and physical interactions of the ARF tumor suppressor with p53 and Mdm2. Proc. Natl. Acad. Sci. USA 95:8292-8297.

7.Kaufman, R., M.V. Davies, L.C. Wasley and D. Michnick. 1991. Improved vectors for stable expression of foreign genes in mammalian cell by use of the untranslated leader sequence from EMC virus. Nucleic Acids Res. 19:44854490.

8.Kaufman, R.J., P. Murtha and M.V. Davies. 1987. Translational efficiency of polycistronic mRNAs and their utilization to express heterologous genes in mammalian cells. EMBO J. 6:187-193.

9.Kim, D.G., H.M. Kang, S.K. Jang and H.-S. Shin. 1992. Construction of a bifunctional mRNA in the mouse by using the internal ribosomal entry site of the encephalomyocarditis virus. Mol. Cell. Biol. 12:3636-3643.

10.Klucher, K.M., M.J. Gerlach and G.Q. Daley. 1997. A novel method to isolate cells with conditional gene expression using fluorescence activated cell sorting (FACS). Nucleic Acids Res. 25:4858-4860.

11.Kobayashi, M., Y. Yamauchi, A. Tanaka and S. Shimamura. 1996. Improved dicistronic mRNA expression vectors for efficient selection of transfectants highly expressing foreign genes. BioTechniques 21:398-402.

12.Miltenyi, S., W. Muller, W. Weichel and A. Radbruch. 1990. High gradient magnetic cell separation with MACS. Cytometry 11:231238.
13.Mosser, D.D., A.W. Caron, L. Bourget, P. Jolicoeur and B. Massie. 1997. Use of a dicistronic expression cassette encoding the green fluorescence protein for the screening and selection of cells expressing inducible gene products. BioTechniques 22:150-161.

14.Rees, S., J. Coote, J. Stables, S. Goodson, S. Harris and M.G. Lee. 1996. Bicistronic vector for the creation of stable mammalian cell lines that predisposes all antibiotic-resistant cells to express recombinant protein. BioTechniques 20:102-110.

15.Scheper, G.C., A.A. Thomas and H.O. Voorma. 1991. The $5^{\prime}$ untranslated region of encephalomyocarditis virus contains a sequence for very efficient binding of eukaryotic initiation factor eIF-2/2B. Biochim. Biophys. Acta 1089:220-226.

16.Seshasayee, D., P. Gaines and D.M. Wojchowski. 1998. GATA-1 dominantly activates a program of erythroid gene expression in factor-dependent myeloid FDCW2 cells. Mol. Cell. Biol. 18:3278-3288.

17.Siebenkotten, G., U. Behrens-Jung and S. Miltenyi. 1998. Employing surface markers for the selection of transfected cells, p. 271281. In D. Rechtenwald and A. Radbruch (Eds.), Cell Separation Methods and Applications. Marcel Dekker, New York.

18.Smarda, J. and J.S. Lipsick. 1993. Dicistronic selection for nuclear proteins in living animal cells. Gene 137:145-149.

19.Stanciu, L.A., J. Shute, S.T. Holgate and R. Djukanovic. 1996. Production of IL-8 and IL4 by positively and negatively selecting CD4+ and CD8+ human T cells following a four-step cell separation method including magnetic cell sorting (MACS). J. Immunol. Methods 189:107-115.

The authors thank Elaine Kunze for expert assistance with FACS and flow cytometry. This work was supported by Grant Nos. NIH HL4491 to D.M.W. and NIH F32 HL 09749 to P.G. Address correspondence to Dr. Don M. Wojchowski, 115 William L. Henning Building, The Pennsylvania State University, University Park, PA 16802, USA.Internet: dmw1@psu.edu

Received 10 June 1998; accepted 26 January 1999.

\section{Peter Gaines and Don M. Wojchowski \\ The Pennsylvania State \\ University \\ University Park, PA, USA}

